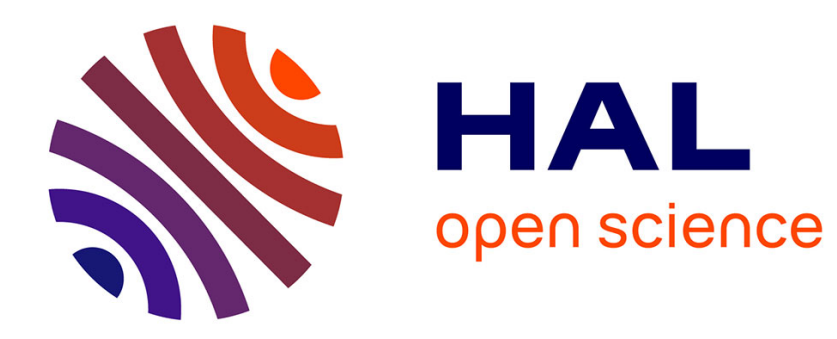

\title{
TRANSITIONS FROM MONOLAYERS TO BULK MATTER
}

\author{
J. Dash
}

\section{To cite this version:}

J. Dash. TRANSITIONS FROM MONOLAYERS TO BULK MATTER. Journal de Physique Colloques, 1977, 38 (C4), pp.C4-201-C4-206. 10.1051/jphyscol:1977430 . jpa-00217146

\section{HAL Id: jpa-00217146 https://hal.science/jpa-00217146}

Submitted on 1 Jan 1977

HAL is a multi-disciplinary open access archive for the deposit and dissemination of scientific research documents, whether they are published or not. The documents may come from teaching and research institutions in France or abroad, or from public or private research centers.
L'archive ouverte pluridisciplinaire HAL, est destinée au dépôt et à la diffusion de documents scientifiques de niveau recherche, publiés ou non, émanant des établissements d'enseignement et de recherche français ou étrangers, des laboratoires publics ou privés. 


\title{
TRANSITIONS FROM MONOLAYERS TO BULK MATTER (*)
}

\author{
J. G. DASH \\ University of Washington, Seattle 98195 , U.S.A.
}

\begin{abstract}
Résumé. - L'équilibre thermodynamique nous permet de décrire la croissance des films en trois classes. En classe I les films sont en couches uniformes à chaque degré de recouvrement de la surface, et ses propriétés deviennent asymptotiques à la phase tridimensionnelle. La classe II commence comme la première mais après une couche uniforme, la croissance change brusquement en formant des petits cristaux ou des gouttes de la phase tridimensionnelle. Les cristaux croissent puis deviennent jointifs en suivant un processus de percolation. Après cette étape, la surface se couvre entièrement par un film épais. Quelques systèmes peuvent changer de classe sous la forme de transitions croisées dans lesquelles les fluctuations d'épaisseur sont très grandes. En classe III la croissance commence par la formation de gouttes.
\end{abstract}

\begin{abstract}
Equilibrium thermodynamics allows us to describe the growth of films in three classes. In class $I$ the films are uniform layers at all coverages, and their properties develop asymptotically to the three-dimensional phase. Class II begins as I but changes abruptly to the growth of crystallites or droplets of the bulk phase. The crystallites grow, and at some definite coverage, develop a continuous connected path of thick film across the surface, in a percolation transition. After this stage there is another special point, at which the surface becomes completely covered by thick film. Some systems can change classes, in crossover transitions which may be marked by large fluctuations in thickness. In class III growth begins with the cluster stage.
\end{abstract}

1. Introduction. - In certain monolayer films one can observe a succession of phases as the coverage is increased: there may be two-dimensional gas, liquid and solid regimes and also one or more registered phases. The course of the second layer may be nearly as intricate, and if the temperature is low enough so that the first layer remains inert through this succession, these phases are essentially attributes of the second layer only. There may be a similar sequence in further deposits, provided that the temperature is so low that adjacent layers can be distinguished from each other, with no natural limit to the progression. In this way the evolution of bulk character might be approached asymptotically by a superposition of monolayer properties.

One is led to ask: is this the only manner of development or are there other modes of film growth ?

It appears that the asymptotic approach is neither the sole nor the dominant mode. In a few systems bulk behavior begins abruptly, with no preceding layer growth. For many more and perhaps most systems, the evolution of bulk character involves a series of different regimes and at least two phase transitions. The first stage of growth is a regime of uniform deposit, up to a thickness which can range

(*) Research supported by National Science Foundation. from a partial monolayer to several layers. This is terminated by a transition to isolated cluster formation. Following an interval of growth the progressive merging of clusters produces a percolation transition which establishes long range connectivity on the surface. After this transition there is a gradual filling in of the thin film regions and then an asymptotic approach to bulk.

The nature of the cluster transition-percolation transition mode of growth and the conditions that favor it or the asymptotic mode can be understood in considerable quantitative detail from the standpoint of classical thermodynamics. Several aspects of the theory are well known while others have been developed only very recently. The present status is surveyed in this paper and some new results are presented.

2. Three modes of growth. - Films progressively built up on uniform substrates take particular styles of growth which can be divided into three classes, as described in the introduction. These three classes were noted many years ago for films floating on liquids [1] and more recently for films on solid surfaces [2]. The thermodynamic basis is perfectly general yet it seems not to be widely known, especially among those working in the field of physisorption. Here we present an outline of the 
theory as in a recent reformulation [3], emphasizing the standpoint of physisorption.

In working with adsorbed films one naturally first thinks of vapor pressure isotherms. They are the most common methods of investigation and they automatically imply growth. Among the experiments on uniform substrates one can distinguish three classes of isotherm, as follows :

Class I. The vapor pressure $P$ approaches the saturated value $P_{0}$ asymptotically with increasing coverage.

Class II. $P$ reaches $P_{0}$ abruptly at finite coverage.

Class III. $P$ reaches $P_{0}$ at zero coverage.

The isotherm classes correspond to the modes of growth; the uniform thickness asymptotic growth mode, the uniform thickness-clustering transition mode, and the direct bulk condensation mode, respectively.

First recall the equation for the contact angle of a macroscopic liquid drop $\left(^{1}\right)$ resting on a solid surface $[1,4]$ :

$$
\cos \theta=\left(\gamma_{s v}-\gamma_{s i}\right) / \gamma_{l v},
$$

where $\gamma$ is the interfacial tension and $s, 1$, v stand for substrate, liquid, and vapor. If the liquid wets the surface completely, the contact angle vanishes, yielding the inequality

$$
\gamma_{\mathrm{sv}}>\gamma_{\mathrm{lv}}+\gamma_{\mathrm{sl}}
$$

Eq. (2) is the criterion for complete wetting, where the surface is covered by a uniform thickness film. The interfacial tension between substrate and vapor is affected by adsorption, which reduces the coefficient of the bare surface by an amount equal to the spreading pressure $\phi$ of the adsorbed film :

$$
\gamma_{\mathrm{sv}}=\gamma_{\mathrm{so}}-\phi
$$

where $\gamma_{s o}$ is the interfacial tension of the substrate in vacuum. Eq. (3) follows from the definition of $\gamma$ and the Gibbs-Duhem relation. For 2 phases in contact the surface tension of the interface is the excess thermodynamic potential per unit area $[4,5]$ :

$$
\gamma \equiv \Omega_{\mathrm{s}} / A
$$

The thermodynamic potential for all systems is the difference between the Helmholtz free energy $F$ and the Gibbs potential $G: \Omega=F-G$. For simple bulk matter $G=F+P V$; in systems with appreciable surface area there is an additional term proportional to $A$. Thus, for the 2 component system the total $\Omega$ can be separated into a part $\Omega_{\mathrm{v}}=-P V$ from the proportionate contributions of the bulk components as if entirely unaffected by the interface, and an

(1) The stability equation holds for the interface between any 3 phases and is not restricted to liquid drops. We use liquid for convenience to mean the condensed phase of the adsorbate. excess contribution $\Omega_{\mathrm{s}} . \Omega_{\mathrm{s}}$ includes all of the changes due to the interface including distortions which may extend over appreciable but limited distances into the bulk phases. If the relaxation lengths are much smaller than the dimensions of the bulk volumes then the effects of the interface will be proportional to the area $A$. Changes in $\gamma$ caused by adsorption can be related to film and vapor properties. Suppose that the interface between a substrate and vacuum has coefficient $\gamma_{\mathrm{so}}=\Omega_{\mathrm{so}} / A$. Now if the vapor is introduced there will be a change in the $\Omega$ of the total system, part of which is proportional to $A$. This change is reflected by a change in the coefficient, $\delta \gamma=\delta \Omega_{\mathrm{s}} / A$. One usually considers the change an attribute of the adsorbed film, although it belongs to the composite substratefilm-vapor ; $\Omega_{\text {film }}=\delta \Omega_{\mathrm{s}}$. It is in principle necessary, especially for thick films, to include a specific volume term as well as an area term in $\Omega_{\mathrm{f}}$, so that

$$
\Omega_{\mathrm{f}}=-P V_{\mathrm{f}}-\phi A \text {. }
$$

In most cases of interest the film is so thin that $P V_{\mathrm{f}}$ can be neglected : the criterion is that the thickness $t \ll \phi / P$. In typical systems the criterion is satisfied at thicknesses up to several hundreds of atomic layers. Under these conditions the substrate-vapor coefficient is simply related to the vacuum value, as given by eq. (3).

Finally, substituting (3) in (2) one has a criterion for complete wetting :

$$
\phi<\phi_{\mathrm{c}}=\gamma_{\mathrm{so}}-\gamma_{\mathrm{lv}}-\gamma_{\mathrm{sl}}
$$

It is important to note that the critical value $\phi_{c}$ involves interfacial coefficients between the bulk phases but does not involve the adsorbed film. Specifically, $\gamma_{\mathrm{si}}$ is the coefficient of the interface between the substrate and the bulk liquid; it does not depend on the form of the transition region, as long as it is relatively thin. There is no need to inspect whether there are any distinct phases or structures in the first few layers of adsorbate next to the substrate, since the coefficient $\gamma_{\mathrm{sl}}$ contains all of the changes in $\Omega$ proportional to the area of the interface. As far as thermodynamics is concerned, it is meaningless to inquire whether condensate rests directly on the substrate or on some very thin intermediate film.

Eq. (6) leads directly to the 3 classes of isotherm. They can be demonstrated by expressing $\phi$ in terms of $P$ via the Gibbs isotherm:

$$
\phi(P)=\int_{0}^{P} \frac{v}{a} \mathrm{~d} P,
$$

where $v$ is the volume/molecule in the vapor and $a$ is the area $/$ molecule in the film. Since $\mathrm{d} \phi / \mathrm{d} P>0, \phi$ is bounded by its value $\phi\left(P_{0}\right)$ at the saturation pressure of the bulk phase. The 3 classes then emerge from the wetting inequality (6), as follows 
Class I. $\phi\left(P_{0}\right)<\phi_{c}$. Complete wetting occurs for all pressures and coverages up to the bulk values. There is a gradual evolution of properties from monolayer to bulk. [Class I isotherms have been obtained with $\mathrm{Kr}[6]$ and $\mathrm{NO}[7]$ on graphite at $77 \mathrm{~K}$.

Class II. $\phi\left(P_{\mathrm{c}}\right)=\phi_{\mathrm{c}}$, where $P_{\mathrm{c}}<P_{0}$. Complete wetting occurs in the range $0<P \leq P_{\mathrm{c}}$; in this range the film is uniform in thickness up to a maximum $t_{\mathrm{c}}$ at $P_{c}$. At $P_{c}$ the contact angle for condensate becomes nonzero and further material goes into the form of clusters or droplets of bulk matter. Between $P_{\mathrm{c}}$ and $P_{0}$ there can be no further deposit of uniform film and no bulk condensate, hence the isotherm is horizontal ; it intersects the $P=P_{0}$ axis at a right angle. The ideal shape is shown in figure 1 . [Class II behavior (i.e. essentially class II behavior, allowing for heterogeneity and other deviations from the ideal model) has been observed in $\mathrm{NO}$ on $\mathrm{CdCl}_{2}, \mathrm{CdBr}_{2}$, and $\mathrm{CdI}_{2}$ near $80 \mathrm{~K}[8]$, in $\mathrm{CH}_{3} \mathrm{Cl}, \mathrm{CH}_{3} \mathrm{~F}$, and $\mathrm{CH}_{3} \mathrm{Br}$ on Cd between $100 \mathrm{~K}$ and $153 \mathrm{~K}$ [9], and in $\mathrm{SF}_{6}$ on $\mathrm{Cu}$ at $143 \mathrm{~K}[10]$.

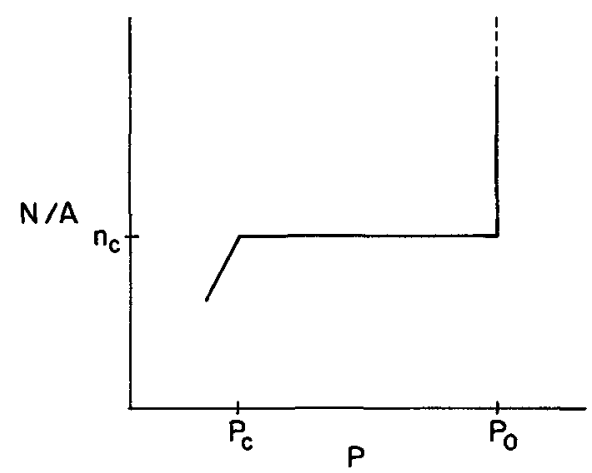

FIG. 1: - Ideal vapor pressure isotherm of a class II film. The substrate is homogeneous, incompressible, and flat on a macroscopic scale, but it may have a distinct atomic texture.

Class III. $\phi_{c}<0$. There can be no adsorption ; the beginning of bulk phase growth is reached without any film phase. [Isotherms of this character, or nearly this character, have been reported for $\mathrm{Kr}$ on $\mathrm{Na}$ and on $\mathrm{Na}_{2} \mathrm{O}$ at $75 \mathrm{~K}$ [11] and $\mathrm{H}_{2} \mathrm{O}$ on graphitized carbon black at $293 \mathrm{~K}$ [12].]

The conditions that govern to which class a system belongs are properties of the bulk phases at each temperature. Since the interfacial tensions vary with $T$ it is possible for a particular adsorbate-substrate combination to fall into one class in a certain temperature range and a different class in another. Indeed, it has been recently observed that $\mathrm{He}^{3}$ on graphite appears to be a class II film at $T \approx 1.2 \mathrm{~K}$ and class I above [13]. A series of isotherms of a film as it crosses from one class to another have the appearance shown in figure 2. One can imagine the boundary being approached from either side, so that the progression

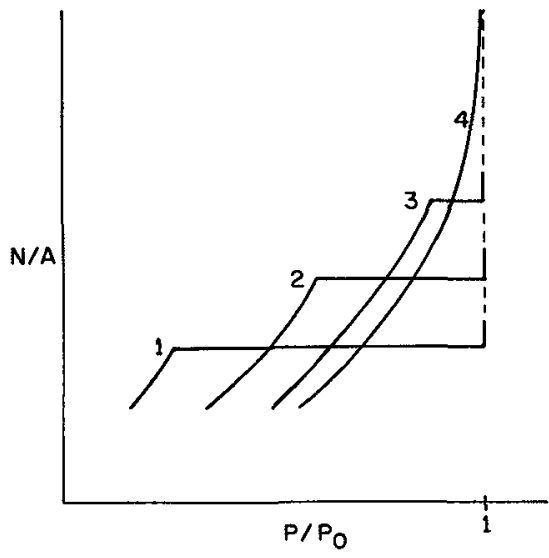

FIG. 2. - A series of isotherms at temperatures in the neighborhood of a class I-class II crossover transition. The sequence may involve either rising or falling temperatures.

can involve either rising or falling temperatures. In the neighborhood of crossover the film ought to be quite unstable with respect to thickness; this should be a special kind of critical point, marked by divergences in the two-dimensional compressibility and other related quantities.

In this discussion we refer to the clusters as liquid droplets, but the theory is not restricted to liquid phase formation. The same theory holds for crystalline deposits, in which case the interfacial coefficients of the appropriate facets must be specified. The stability conditions are different for individual orientations of crystallites, and there will be one particular set of exposures for which the condition for finite contact angle is reached first. It is this combination that sets $t_{c}$, and the subsequent growth is epitaxial with the corresponding facets exposed.

3. Nucleation and early growth : the clustering transition. - In their section on nucleation of bulk phase droplets from a saturated vapor, Landau and Lifshitz state [4]: If the saturated vapor is in contact with a solid surface (the walls of a vessel) which is completely wetted by the liquid, then the vapor will condense directly on the solid surface without any nuclei appearing. The creation of a liquid film on the solid surface does not, in this case, require any work to be performed to create the surface and thus the existence of a metastable phase is impossible.

This statement holds without qualification with regard to class I films, but it has to be inspected carefully in the case of class II films. For in these systems the complete wetting film at $a>a_{\mathrm{c}}$ is a phase distinct from the bulk liquid and hence the formation of droplets does require the creation of new interfaces. The creation process involves net work per unit area

$$
\Delta \gamma=\gamma_{\mathrm{sv}}+\gamma_{\mathrm{sl}}-\gamma_{\mathrm{sv}}
$$


where $\gamma_{\mathrm{sv}}$ is the coefficient at $P=P_{0}$. Since $\gamma_{\mathrm{sv}}+\gamma_{\mathrm{sl}}=\gamma_{\mathrm{sv}}\left(P_{\mathrm{c}}\right)$ and $\gamma_{\mathrm{sv}}=\gamma_{\mathrm{so}}-\phi$, eq. (8) together with (7) and the ideal gas approximation for the vapor yields

$$
\Delta \gamma=\phi\left(P_{0}\right)-\phi\left(P_{\mathrm{c}}\right)=n_{\mathrm{c}} k T \ln \left(P_{\mathrm{o}} / P_{\mathrm{c}}\right),
$$

where $n_{\mathrm{c}}$ is the critical coverage $(n=1 / a)$.

Now if $P_{\mathrm{c}}<P_{\mathrm{o}}$ there is a finite coefficient between the film-covered substrate and the bulk condensate, and the problem of nucleation on the covered surface is essentially the same as on a bare surface.

In ideal systems of class II or III films the formation of bulk phase is guarded by a high nucleation barrier. The barrier is due to the fact that the cohesive energy of the condensed phase is smaller in clusters than in the bulk, so that the clusters have larger vapor pressures. Hence it is necessary to supersaturate the vapor in order to nucleate condensation, as illustrated in figure $3 a$. Even with appreciable supersaturation pressures the nucleation times can be astronomically long. The probability of appearance of a droplet of volume $V$ at slight excess pressure $\delta P$ is [4]

$$
W \sim \exp \left[-\frac{16 \pi \gamma^{3} V^{2} P^{2}}{3(k T)^{3}(\delta P)^{2}}\right]
$$

In the film case the effective surface tension is the quantity $\Delta \gamma$ given by eq. (9), and the droplets are not spherical, which introduces a geometrical factor ; otherwise the probability has the same form. Typical probabilities according to [10] are extremely
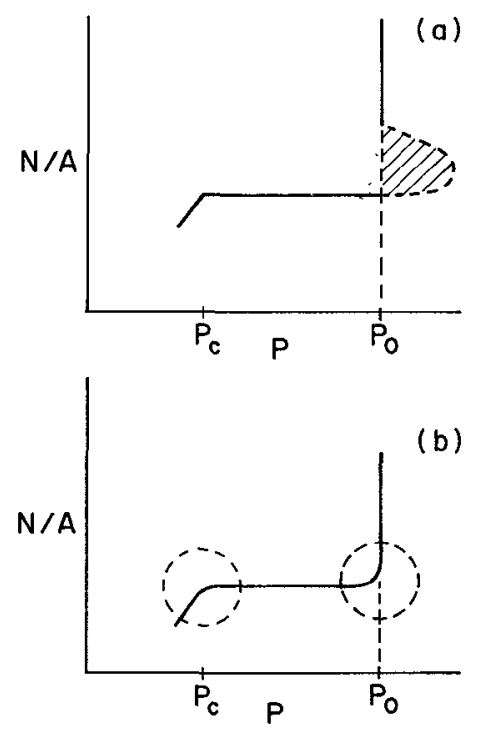

FIG. 3. - Class II isotherms near the initiation of cluster growth. (a) On an ideal surface the end of the uniform layer regime is abrupt at $P_{c}$, and the beginning of cluster growth is guarded by a large nucleation barrier. On real surfaces both points are blurred by heterogeneity. The end of layer growth occurs at different pressures on different regions of the surface. Cluster growth can be nucleated at imperfections, reducing or completely eliminating the barrier. small. For example, if $\Delta \gamma=1 \mathrm{erg} / \mathrm{cm}^{2}, T=100 \mathrm{~K}$, $V=(10 \AA)^{3}$, and $\delta P / P=0.1, W \sim \exp \left(-6 \times 10^{5}\right)$.

However, on real surfaces there always are imperfections which can reduce or entirely remove the nucleation barrier. At kink sites and the inside corners of growth or cleavage steps the adsorption energy is greater than on the large facets, and at these places the adsorbed film is thicker. As $P$ is increased there is a tendency for the local adsorption to fill up the corners and pits, because their concave surfaces have lower equilibrium pressure than flat regions. This preferential thickening can be so pronounced that bulk formation occurs at the special locations before $P$ reaches $P_{0}$, in which case there is no longer any nucleation barrier for the system as a whole, and clusters can just grow outward continuously from the nucleation sites into the uniform regions. The number and nature of the nucleation sites affect the course of $P$ between $P_{c}$ and $P_{0}$; for instance, they can cause the intersection with the $P_{0}$ axis to be rounded instead of abrupt, as in figure $3 b$, and as in experimental isotherms.

4. Droplet growth, percolation, and evolution to thick film. - After the nucleation stage further deposition causes each cluster to grow. When the clusters are very small they are markedly flattened by the substrate field instead of having the spherical cap shapes of large droplets. The distorted shapes can be derived analytically [3]. A small droplet of lateral dimension $l$ on a substrate with interaction constant $\alpha$ (attractive energy at $t$ atomic diameters $=\alpha / t^{3}$ ) is predicted to have mean thickness $\bar{h}$ :

$$
\bar{h} \sim\left(\alpha l / \gamma_{\mathrm{lv}}\right)^{1 / 3}
$$

Typical thicknesses are much less than lateral dimensions. As an example, eq. (11) applied to $\mathrm{N}_{2}$ films on graphite predicts that for $l=-100 \AA$, $\bar{h} \sim 20 \AA$, while for $l=500 \AA, \bar{h} \sim 35 \AA$. Therefore in this range of magnitudes the droplets increase in size primarily by lateral growth, with very modest increases in thickness.

During droplet growth the thin uniform film regions remain at the same critical thickness $t_{\mathrm{c}}$, hence all of the adsorbate added beyond the critical quantity $N_{\mathrm{c}}$ goes into the growing droplets. In this growth regime the vapor pressure isotherm has no distinguishing features, but other thermodynamic quantities can indicate 2 distinct phases in the film. The signature of phase coexistence in any thermodynamic system is a linear dependence of extensive properties on the quantity of substance. The heat capacity, for example, has the functional dependence on adsorbate quantity $N$ and temperature $T$, in a system of fixed $A$ and $V$,

$$
C(N, T)=N f(T)+g(T),
$$


in any region of 2-phase equilibrium [14]. Such behavior has been seen in the heat capacity of $\mathrm{N}_{2}$ films [15] and in the inelastic neutron scattering of $\mathrm{He}^{4}$ films [16]; in both cases, we believe, it is due to clustering.

Since the material added beyond nucleation goes completely into the new phase and the thickness of the droplets grows very slowly with lateral size, the area of substrate covered by clusters increases nearly proportional to $\left(N-N_{c}\right)$. Taking the thickness dependence into account according to eq. (11), the thick film area increases as $\left(N-N_{c}\right)^{6 / 7}$.

In the stage of droplet growth the contact angle between the droplets and the film covered surface (measured some distance above the substrate in the field-free region) contains some information about the uniform regions; combining eqs. (1), (6) and (9), we find

$$
\cos \theta=1-\left(\frac{n_{\mathrm{c}} k T}{\gamma_{\mathrm{lv}}}\right) \ln \left(P_{\mathrm{o}} / P_{\mathrm{c}}\right) .
$$

When the droplets grow far enough (which depends on the spatial distribution of nucleating sites), neighboring droplets coalesce, forming irregularly shaped patches of thick film. As growth continues the average patch length increases and connections multiply. At some stage there is a continuous path of thick film across the entire width of substrate. The statistics of the connection process and the establishment of long range connectivity can be formulated in terms of percolation theory. A class II or III film may exhibit a kind of twodimensional percolation transition. Recent measurements of the electrical conductivity of a $\mathrm{Bi}$ film show that there is an abrupt drop in resistance when the fractional area covered by thick film reaches about 0.67 ; this result and the exponent of the variation of connectivity with coverage are consistent with predictions of theory [17].

Connectivity is not an important quantity in typical physisorbed films, but in $\mathrm{He}^{4}$ below its $\lambda$ point the long range connectivity can be readily measured via anomalous transport coefficients. It is argued that superfluid onset in $\mathrm{He}^{4}$ films is a special kind of percolation transition, in which connectivity is established through phase coherence between the order parameters of individual droplets rather than the actual merging of droplets [3].

Beyond the percolation transition the thick film patches continue to grow at the expense of the thin regions. At some stage in this process the thin regions are completely gone and the surface is covered by a thick film. It appears that there may be a fairly definite point at which this condition is reached. This thick film point has coverage $n_{\text {th }}$ :

$$
n_{\mathrm{th}}-n_{\mathrm{c}} \simeq\left(\alpha / \gamma_{\mathrm{lv}}\right)^{1 / 3} n_{\mathrm{n}}^{-1 / 6} v_{1}^{-1} .
$$

$n_{\mathrm{n}}$ is the areal density of nucleating sites, which we here assume to be uniformly distributed. The actual coverage at the thick film point and the sharpness of this transition obviously depend on the uniformity of the distribution, but the dependence is quite weak. To illustrate, the predicted thick film point for $\mathrm{He}^{4}$ on graphite at low $T$ occurs at $n_{\text {th }}-n_{\mathrm{c}} \simeq 7 \times 10^{15}$ (equivalent to $\sim 20$ liquid layers) if the mean separation between nucleating sites is $1000 \AA$, and about half of this coverage if the separation is only $100 \AA$. Estimates for $\mathrm{N}_{2}$ on graphite are comparable.

After the thick film point further growth is asymptotic to bulk phase. Some properties of the films in this regime may differ very little from bulk while others may remain significantly different. For example, a superconducting film appears twodimensional as long as its thickness is less than the coherence length, which may be several hundred $\AA$, while its normal conductivity above $T_{c}$ resembles bulk metal at thicknesses $\sim 10 \AA$.

5. Discussion. - Various features of the theoretical behavior have been observed in experimental films, but data on well characterized substrates are incomplete. There are many measurements of cluster onset and growth in metal and strongly cohesive films, but little information on their critical thicknesses, the coverages at the percolation and thick film transitions, or the temperatures of crossover transitions. Van der Waals films should be particularly convenient subjects for study, using currently available experimental techniques.

Note added in proof. Several recent developments and corrections should be noted. A crossover transition between classes I and II appears to occur in the system ethane on exfoliated graphite between $88 \mathrm{~K}$ and $97 \mathrm{~K}$, according to isotherms observed by REGNIER J. (Thesis, Univ. of Nancy, 1976). A theory of superfluid onset in $\mathrm{He}^{4}$ films, involving clustering and Josephson weak links between droplets, has been proposed (HUBERMAN B.A. and DASH J.G., to be published). An alternative theory of film growth, also based on equilibrium thermodynamics, has been developed by R.E. Peierls (private communication). Peierls' theory yields the same wetting criterion as eq. (6) and the classification of growth modes. However, an error in the author's treatment has been uncovered by Peierls' analysis. The shape of a class II isotherm may be more gradual than as shown in figure 1 , and the pressure may reach the $P_{0}$ axis at a finite angle different from 90 degrees. Peierls has also shown (private communication) that an improved approximation can be given for the aspect ratio of droplet width to thickness. Instead of the eq. (13) the dependence of mean thickness on lateral dimension becomes as the square root. There are consequent changes in the dependence of thick film area on coverage and the thick film point. Details of the improved calculations will be published soon. 


\section{References}

[1] Harkins, W. D., The Physical Chemistry of Surface Films (Reinhold Publ. Co. N.Y.) 1952.

[2] BAUER, E., Z. Kristallogr. 110 (1958) 395.

[3] Dash, J. G., Phys. Rev. B 15 (1977) 3136.

[4] Landau, L. D. and Lifshitz, E. M., Statistical Physics (Pergamon Press Ltd, London and Paris) 1958.

[5] GibBs, J. W., Scientific Papers (Longmans, Green Ltd., London) 1906.

[6] Thomy, A. and Duval, X., J. Chim. Phys. 67 (1970) 286.

[7] Matecki, M., Thomy, A. and Duval, X., Comptes rendus 273 (1971) 1.

[8] Matecki, M., Thomy, A. and Duval, X., J. Chim. Phys. 71 (1974) 1484

[9] Genot, B., J. Chim. Phys. 70 (1973) 1565.

[10] Genot, B. and Duval, X., J. Chim. Phys. 70 (1973) 134
[11] Pierotti, R. A. and Halsey, G. D., J. Phys. Chem. 63 (1959) 680.

[12] Avgul, N. N., Berezin, G. I., Kisilev, A. V., and LyGina, I. A., Izv. Akad. Nauk SSSR, Otd. Khim. Nauk 2 (1961) 205.

[13] VAN SCIVER, S. and VILChES, O. E., Phys. Lett. 55A (1975) 191.

[14] DASH, J. G., Films on Solid Surfaces (Academic Press, N.Y.) 1975 .

[15] Chung, T. T. and Dash, J. G., J. Chem. Phys. 64 (1976) 1855.

[16] Carneiro, K., Ellenson, W., Passell, L., Mctague, J. P. and TAUb, H., Phys. Rev. Lett. 37 (1976) 1695.

[17] Liang, N. T., Shan, Y., and WANG, S. Y., Phys. Rev. Lett. 37 (1976) 526

\section{DISCUSSION}

F. A. PUTNAM. - Wade has taken adsorption isotherm measurements on flat plates where he has been able to explore the metastable region at higher pressures than $P_{0}$.

F. A. PUTNAM. - It appears that a critical point may be found between type I and type II behavior. Do you have any information on whether such a critical point exists, and if so, what its universality class is ?

J. G. DASH. - There should be several types of critical points, corresponding to the different types of growth crossovers (e.g. between I and II, between II and III, or on rising or falling temperature), but there have been no observations that I am aware of. I believe that these transitions will resemble the roughening transitions of crystalline surfaces, but I have no information on their universality class.

W. STEELE. - The surface of capillary waves cause adsorbed films to undergo fluctuations in position, just as in the bulk droplets. Would the inclusion of these fluctuations in your theory have an appreciate effect on the results?

J. G. DASH. - The theory automatically includes all surface excitations such as capillary waves in the single coefficient of surface tension for each interface. Capillary waves can certainly affect the behavior of liquid surfaces, but to the extent that we can accept the thermodynamic coefficients as fundamental variables of the system, it is not necessary to inspect the specific effect of any class of excitations such as capillary waves.
J. P. McTague. - There are recent neutron experiments at Brookhaven studying the roton spectrum of ${ }^{4} \mathrm{He}$ at $T=1.3 \mathrm{~K}$, on Spheron. Over the range $4 \leqslant n \leqslant 10$, where $n$ is the total number of adsorbed layers, the width of the roton is of the form $\Gamma \sim(n-\delta)^{-1}$, where $\delta \sim 3.5-3.8$ layers. The most straight forward interpretation of these results is that the coverage is uniform within this range, rather than puddling.

J. G. DASH. - There are many long-standing difficulties with a uniform film model of $\mathrm{He}^{4}$ adsorption, especially with regard to the onset of superfluidity. The droplet-percolation model seems to answer most of the puzzles, even to the details of the relation between thickness and superfluid onset temperature. [For detailed reviews and discussions, see Dash, J. G., Physics Reports (to be published) and Huberman, P. A. and Dash, J. G., Phys. Rev. Lett. (to be published).] As far as the roton width is concerned, it seems that this new measurement is quite consistent with droplet formation. If the line does indeed remain Lorentzian, it implies that the roton lifetime is shortened (linearly) as the droplet size is reduced, which seems a most reasonable result. If there is a certain degree of inhomogeneous broadening, there would be a degree of gaussian character introduced. Perhaps both occur within the experimental accuracy. One way of testing the theory would be by trying the same experiment on different qualities of graphite : on more uniform substrates there will be fewer but larger droplets, hence the broadening will be reduced. 